\title{
Abnormalities in Mitochondrial Structure in Cells from Patients with Bipolar Disorder
}

Anne M. Cataldo, ${ }^{\star \dagger \ddagger}$ Donna L. McPhie, ${ }^{* \neq}$ Nicholas T. Lange, ${ }^{* £ \S}$ Steven Punzell, ${ }^{*}$ Sarah Elmiligy, ${ }^{\dagger \ddagger}$ Nancy Z. Ye, ${ }^{\dagger \ddagger}$ Michael P. Froimowitz, ${ }^{\star \S}$ Linda C. Hassinger ${ }^{\dagger}$ Emily B. Menesale, ${ }^{\dagger \ddagger}$ Laura W. Sargent, ${ }^{\dagger \ddagger}$ David J. Logan, ${ }^{\text {"T }}$ Anne E. Carpenter, ${ }^{\text {Tा }}$ and Bruce M. Cohen ${ }^{\star \ddagger}$

From the Department of Psychiatry," Harvard Medical School, Boston; the Laboratory of Molecular Neuropathology, ${ }^{\dagger}$ the Neurostatistics Laboratory, ${ }^{\S}$ and the Molecular Pharmacology Laboratory, ${ }^{\ddagger}$ Mailman Research Center and Frazier Research Institute, McLean Hospital, Belmont; and the Broad Institute of Harvard and MIT, ${ }^{\text {पा }}$ Cambridge Massachusetts

Postmortem, genetic, brain imaging, and peripheral cell studies all support decreased mitochondrial activity as a factor in the manifestation of Bipolar Disorder (BD). Because abnormal mitochondrial morphology is often linked to altered energy metabolism, we investigated whether changes in mitochondrial structure were present in brain and peripheral cells of patients with BD. Mitochondria from patients with BD exhibited size and distributional abnormalities compared with psychiatrically-healthy age-matched controls. Specifically, in brain, individual mitochondria profiles had significantly smaller areas, on average, in BD samples $(P=$ 0.03). In peripheral cells, mitochondria in BD samples were concentrated proportionately more within the perinuclear region than in distal processes $(P=0.0008)$. These mitochondrial changes did not appear to be correlated with exposure to lithium. Also, these abnormalities in brain and peripheral cells were independent of substantial changes in the actin or tubulin cytoskeleton with which mitochondria interact. The observed changes in mitochondrial size and distribution may be linked to energy deficits and, therefore, may have consequences for cell plasticity, resilience, and survival in patients with BD, especially in brain, which has a highenergy requirement. The findings may have implications for diagnosis, if they are specific to $\mathrm{BD}$, and for treatment, if they provide clues as to the underlying pathophysiology of BD. (Am J Pathol 2010, 177:575-585; DOI: 10.2353/ajpath.2010.081068)
Bipolar disorder (BD) is a major and relatively common psychiatric illness characterized by recurrent manic and depressive episodes. Risk for BD is largely determined by genetic factors, although specific genes have not been consistently identified, ${ }^{1-3}$ nor is it clear why these risk genes would predominantly affect brain function. One clue may be that the brain requires an unusually high level of energy production to maintain ionic gradients and metabolize and detoxify neurotransmitters. Under normal circumstances mitochondria generate most of this energy by oxidative phosphorylation. A number of postmortem, ${ }^{4-8}$ imaging, ${ }^{9,10}$ and genetic ${ }^{4-8,11-15}$ studies suggest that mitochondrial dysfunction may play a key role in the pathophysiology of BD. For example, alterations in the expression patterns of genes associated with mitochondrial activity and in respiratory chain components are found both in brain and lymphocytes from patients with BD. ${ }^{5,14}$ Concordantly, brain imaging studies observe increased lactate, a product of glycolysis rather than oxidative phosphorylation, and decreased phosphocreatine, a high energy storage molecule, in patients with $\mathrm{BD}$, suggesting mitochondrial dysfunction.

Mitochondrial function is closely linked both to mitochondrial shape and to the intracellular distribution of mitochondria, with both shape (size) and distribution differing by cell type and, within cells, changing to reflect alterations in cell activity associated with variation in normal energy demands or abnormal demands related to illness. ${ }^{16-18}$ Also, changes in mitochondrial morphology and distribution appear to be closely linked to diverse cellular events, such as differentiation, aging, and apoptosis. ${ }^{17,19-23}$ Similarly, not only mitochondrial function, but qualitative and quantitative changes in the mitochondrial reticulum have also been associated with mutations

Supported by David Barlow, The Engelhard Foundation, Jeanne and Sanford Robertson, and Stuart and Suzanne Steele (B.M.C.), and by National Institutes of Health grants NS37483 and MH60450 (N.T.L.).

Accepted for publication April 21, 2010.

Author Anne Cataldo passed away in April 2009

Supplemental material for this article can be found on http://ajp. amjpathol.org

Address reprint requests to Donna L. McPhie, Ph.D., Mailman Research Center, McLean Hospital, 115 Mill Street, Belmont MA 02478. E-mail: mcphie@helix.mgh.harvard.edu. 
Table 1. Demographic Data for Postmortem Brain Tissue

\begin{tabular}{|c|c|c|c|c|c|c|c|}
\hline Case & Diagnosis & Sex & Race & Age & PMI(h) & Cause of death & Psychotropic medication use at time of death \\
\hline AN01357 & Control & M & $\mathrm{U}$ & $41-50$ & 18.3 & Myocardial infarction & None \\
\hline AN03375 & Control & M & $U$ & $71-80$ & 20.5 & Heart attack & None \\
\hline AN11618 & Control & M & U & $61-70$ & 25.6 & Heart attack & None \\
\hline AN10411 & Control & $\mathrm{M}$ & U & $31-40$ & 25.7 & Unknown & None \\
\hline AN03422 & Control & M & U & $41-50$ & 24.1 & Unknown & None \\
\hline AN15583 & Control & $\mathrm{M}$ & U & $71-80$ & 20.5 & Unknown & None \\
\hline AN13379 & Control & M & W & $81-90$ & 7.42 & Cancer & None \\
\hline AN04734 & Control & $\mathrm{F}$ & U & $71-80$ & 23.9 & Cancer & None \\
\hline AN14911 & Control & $\mathrm{F}$ & W & $61-70$ & 22.5 & Cancer & None \\
\hline AN04851 & Control & M & U & $61-70$ & 22.33 & Heart attack & None \\
\hline AN18981 & Bipolar disorder & M & W & $31-40$ & 30.75 & Suicide by hanging & $\begin{array}{l}\text { Haloperidol, risperidone lorazepam, quetiapine, } \\
\text { gabapentin, Citalopram, temazepam, } \\
\text { chlordiazepoxide }\end{array}$ \\
\hline AN13972 & Bipolar disorder & $\mathrm{F}$ & W & $71-80$ & 21.46 & Heart attack & Risperidone, lorazepam, olanzapine, gabapentin \\
\hline AN01679 & Bipolar disorder & $\mathrm{M}$ & U & $61-70$ & 27.17 & Respiratory failure & Lithium, divalproex, quetiapine, fluphenazine \\
\hline AN11117 & Bipolar disorder & $\mathrm{F}$ & W & $71-80$ & 29.33 & Unknown & $\begin{array}{l}\text { Risperidone, divalproex, duloxetine, haloperidol, } \\
\text { Quetiapine, lorazepam, }\end{array}$ \\
\hline AN07779 & Bipolar disorder & M & W & $80+$ & 15.5 & Unknown & Lithium, divalproex, donepezil \\
\hline AN05114 & Bipolar disorder & M & $U$ & $80+$ & 17.5 & Unknown & Divalproex, paroxetine \\
\hline AN08409 & Bipolar disorder & M & W & $41-50$ & 30.5 & Heart attack & Lithium \\
\hline AN04190 & Bipolar disorder & M & W & $31-40$ & 22 & Suicide & Divalproex, paroxetine, clonapin, olanzapine \\
\hline AN14219 & Bipolar disorder & M & W & $71-80$ & 27.66 & Unknown & $\begin{array}{l}\text { Lithium, Trazodone, Allopurinol, Digoxin, } \\
\text { Methylprednisolone, Aspirin }\end{array}$ \\
\hline AN18651 & Bipolar disorder & M & W & $81-90$ & 5.02 & Unknown & Unknown \\
\hline
\end{tabular}

$M$, male; F, female; $U$, undetermined; $W$, white.

of mitochondrial DNA ${ }^{24}$ or of nuclear-encoded genes linked to mitochondrially based oxidative phosphorylation (OXPHOS). ${ }^{25}$

Despite the various findings suggesting mitochondrial dysfunction in BD and separate evidence that altered mitochondrial morphology is crucially linked to altered energy metabolism, mitochondrial structure and distribution have not previously been studied in BD. For these reasons, we examined the size (shape) and distribution of mitochondria in $\mathrm{BD}$ using postmortem human brain and primary peripheral cells, both skin fibroblasts and blood lymphocytes, from individuals with BD and healthy comparison subjects.

\section{Materials and Methods}

\section{Tissue and Primary Cell Lines}

Postmortem tissue was obtained from prefrontal cortex (PFC) from ten individuals with BD Type 1, (ages 30-80 years) and an equal number of age-matched controls with no known history of psychiatric illness (Table 1). All tissue was procured from the Harvard Brain Tissue Resource Center (HBTRC), McLean Hospital, Belmont, MA and had previously been fixed in 10\% formalin under standard procedures listed on the HBTRC website.

Fibroblast lines, comprising 8 BD and 8 age-matched psychiatrically-well comparison (control) subjects (ages 20-65 years) (Table 2), and transformed lymphocyte (lymphoblast) lines, comprising $6 \mathrm{BD}$ and 6 age-matched controls, were obtained from the Coriell Institute of Medical Research, Camden, NJ. Fibroblast cell lines were grown in Minimal Essential Media plus 15\% fetal bovine serum (FBS) and 1\% glutamine, penicillin, and strepto- mycin in $5 \% \mathrm{CO}_{2}$. Lymphocytes were grown in RPMI plus $15 \% \mathrm{FBS}$ and $1 \%$ glutamine, penicillin, and streptomycin in $5 \% \mathrm{CO}_{2}$. All cell culture media was from Invitrogen (Invitrogen Corporation, Carlsbad, CA). Media was changed every other day. Cell lines were used between passages 3 and 14 .

\section{Light Microscopic Probes}

Fibroblasts were plated at a density of $2.5 \times 10^{4}$ cells per coverslip. MitoTracker-M7510, $125 \mathrm{nmol} / \mathrm{L}$, (Invitrogen Corporation, Carlsbad CA), was used to visualize mitochondria according to manufacturer's instructions. Cytoskeletal integrity was examined by immunocytochemistry (see below) using an antibody to tubulin $\beta$ I and II (Sigma-Aldrich Corp., St. Louis, MO and Cell Signaling, Danvers, MA) or with fluorescent phalloidin (Invitrogen Corporation, Carlsbad, CA). Cultured cells labeled with fluorescent M7510 were fixed with 4\% paraformaldehyde at $37^{\circ} \mathrm{C}$, washed two times with PBS, and treated with phalloidin (1:8000) for 20 minutes at room temperature for visualization of the actin cytoskeleton (actin filaments) and then mounted in gel mount that contained either 4', 6-diamidino-2-phenylindole (DAPI; $200 \mathrm{nmol} / \mathrm{L})$ or bisbenzimide (Hoechst 33258; $5 \mu \mathrm{g} / \mathrm{ml}$ ) (both from SigmaAldrich Corp., St. Louis, MO) for identification of the nucleus and as a marker of nuclear changes indicative of apoptosis. ${ }^{26}$

\section{Immunocytochemistry of Cytochrome $C$ in Fibroblasts}

Cells were grown on glass coverslips and fixed as detailed above. Samples were washed three times with 
Table 2. Demographic Information for Fibroblast Lines Used

\begin{tabular}{|c|c|c|c|c|c|}
\hline Age & Sex & Diagnosis & Location biopsy & Family & Medications \\
\hline \multicolumn{6}{|l|}{ Control } \\
\hline 20 & M & Not clinically affected & Leg & None & None \\
\hline 61 & $\mathrm{M}$ & Not clinically affected & $\mathrm{N} / \mathrm{A}$ & None & None \\
\hline 30 & $\mathrm{~F}$ & Not clinically affected & $\begin{array}{l}\text { The mesial aspect of the } \\
\text { mid-upper left arm }\end{array}$ & None & None \\
\hline 23 & M & Not clinically affected & $\mathrm{N} / \mathrm{A}$ & None & None \\
\hline 27 & $\mathrm{M}$ & Not clinically affected & $\mathrm{N} / \mathrm{A}$ & None & None \\
\hline 29 & M & Not clinically affected & $\mathrm{N} / \mathrm{A}$ & None & None \\
\hline 25 & $\mathrm{~F}$ & Not clinically affected & Left forearm & None & None \\
\hline 52 & $\mathrm{~F}$ & Not clinically affected & $\begin{array}{l}\text { The mesial aspect of the } \\
\text { mid-upper left arm }\end{array}$ & None & None \\
\hline \multicolumn{6}{|l|}{ Bipolar disorder } \\
\hline 23 & $\mathrm{~F}$ & $\begin{array}{l}\text { BD onset at age } 18 \\
\text { alcoholic, drug abuse }\end{array}$ & Posterior iliac crest & 3 affected in family & Lithium \\
\hline 20 & M & $\begin{array}{l}\text { BD onset at age } 17 \text { drug } \\
\text { abuse }\end{array}$ & Posterior iliac crest & 6 affected in family & Lithium \\
\hline 28 & $\mathrm{~F}$ & $\mathrm{BD}$ & $\mathrm{N} / \mathrm{A}$ & 5 affected in family & $\begin{array}{l}\text { Lithium; Haldol; Cogentin } \\
\text { and Thorazine }\end{array}$ \\
\hline 24 & M & $\begin{array}{l}\text { BD medical disorder with } \\
\text { seizures; alcoholism at } \\
\text { age } 15 \text { depression at } \\
\text { age } 23\end{array}$ & N/A & $\begin{array}{l}\text { Mother and brother } \\
\text { have BD }\end{array}$ & N/A \\
\hline 22 & M & BD onset at age 20 & $N / A$ & Father has BD & Lithium hospitalized twice \\
\hline 65 & M & BD onset at age 18 & $\mathrm{~N} / \mathrm{A}$ & 6 affected in family & None \\
\hline 26 & $\mathrm{M}$ & $\mathrm{BD}$ onset at age 13 & $\mathrm{~N} / \mathrm{A}$ & 4 affected in family & $\begin{array}{l}\text { Lithium and haldol } \\
\text { hospitalized }\end{array}$ \\
\hline 51 & $\mathrm{~F}$ & $\mathrm{BD}$ onset at age 31 & $\mathrm{~N} / \mathrm{A}$ & $\begin{array}{l}2 \text { similarly affected sons } \\
\text { and } 1 \text { daughter }\end{array}$ & Lithium \\
\hline
\end{tabular}

BD indicates bipolar disorder; $M$, male; $F$, female; N/A, not available.

PBS, washed in dilution buffer (PBS $+2 \%$ BSA $+1 \%$ normal serum $+0.1 \%$ Triton-X100), and incubated with $0.5 \%$ SDS in PBS to facilitate antigen retrieval. After blocking in $10 \%$ serum, the cells were incubated in primary antibody to cytochrome C (1:2000) (Mitosciences, Eugene OR) overnight at room temperature with gentle rocking, then washed three times with dilution buffer, followed by incubation in fluorescent-conjugated secondary antibody for 2-3 hours at room temperature. To visualize the tubulin cytoskeleton, the cells were then incubated overnight with an antibody to $\beta$ tubulin directly conjugated to Alexa 555 (Cell Signaling, Danvers MA). Coverslips were then mounted and examined using a Zeiss epifluorescent microscope.

\section{Electron Microscopy}

Cells and vibratome-sectioned brain tissue were processed following standard EM protocols. All chemicals were from Ted Pella (Reading, PA). Briefly, tissue and cells were postfixed in $1 \% \mathrm{OsO}_{4}$, dehydrated in a series of alcohols up to absolute alcohol and propylene oxide and embedded in Epon. Blocks were sectioned, stained, and examined on a JEOL 1200EX microscope.

\section{Measurement of Mitochondrial Area in EM Images}

A two-dimensional analysis using Image Pro Analyzer 6.3 (MediaCybernetics, Bethesda, MD) was used to quantify mitochondrial area from $10 \mathrm{BD}$ and 10 control postmor- tem brain samples. Briefly, mitochondrial areas of 300 mitochondrial profiles were measured from digital images of cells from postmortem PFC at $\times 10,000$ magnification. Ultrastructural images from thirty fibroblasts were obtained at random (magnification $\times 10,000$ ) from each of seven healthy controls and eight $\mathrm{BD}$ cell lines (in total, 210 control fibroblasts and 240 BD fibroblasts). For each line of fibroblasts, 150 mitochondrial profiles in digital images from electron micrographs were measured with Image Pro 6.3 as above. Additionally the number of ring and cup shaped mitochondrial profiles were counted for each fibroblast sample. (Cup shaped was defined as being at least one half of a ring shape.) In both of the above data sets, the person doing the measurement was blind to the diagnostic condition of the sample.

\section{Analysis of Cytochrome C Immunohistochemistry of Human Prefrontal Cortex}

Vibratome sections of fixed PFC tissue from a subset of our brain samples (six BD and six Control subjects) were boiled in TBS for 3 minutes to facilitate antigen exposure and then rinsed three times in TBS. Endogenous peroxidase activity was blocked by incubating sections in $0.3 \%$ $\mathrm{H}_{2} \mathrm{O}_{2}$ in methanol for 30 minutes, and then sections were washed three times in dilution buffer. Sections were then blocked in $20 \%$ goat serum and incubated in anti cytochrome C antibody (Calbiochem, Gibbstown NJ) at a dilution of 15:1000 in dilution buffer. After washes, sections were incubated in biotinylated secondary antibody 
for 30 minutes and ABC (Vector Labs, Burlingame, CA) for 1 hour. Staining was visualized with $D A B$ and then sections were mounted on slides, dehydrated, and cleared with xylene. Eighty images of DAB-positive cells were taken in bright field at $\times 1000$. Before line profile analysis images were processed with Adobe Photoshop using the autocolor and subsequent grayscale commands. Grayscale images were analyzed with line profile analysis using Image Pro Analyzer 6.3 by drawing a line through the longest axis of the cell. Analysis was blind to the diagnostic condition of the sample. Line profile transects were centered and scaled to have mean 0 and variance 1 , interpolated across a common median length across all brains and cells and smoothed objectively to remove high-frequency noise. ${ }^{27}$ Transects were then averaged across all cells sampled from each brain separately, and the result was averaged within groups for between-group comparison and assessed by a Kolmogorov-Smirnov test.

\section{Morphological Analysis of Mitochondrial Distribution Using Digital Images}

We quantified and analyzed mitochondrial density differences between fibroblasts obtained antemortem from the $\mathrm{BD}$ and control groups plated at a density of $2.5 \times 10^{4}$ cells per coverslip. Five random fields of view at $\times 630$ magnification were obtained from 24 to 41 cells from each of $n=8 \mathrm{BD}$ subjects (total $\mathrm{BD}$ cells analyzed $=$ 231) and in 20 to 40 cells from each of $n=8$ healthy age-matched control subjects (total healthy cells analyzed $=251$ ). The number of cells per image varied across subjects from 2 to 11 . For each cell in each digital image, inner and outer cytoplasmic boundaries were traced manually. The inner boundary coincided with the nuclear envelope as determined by DAPI or Hoechst staining, and the outer boundary coincided with the external cell membrane of the fibroblast as determined by phalloidin staining of actin. No overlap was allowed between outer boundaries for different cells. For each cell, a set of concentric, equally spaced, scaled copies of the nuclear envelope were then aligned with the centroid of the envelope to segment the entire cytoplasmic area into expanding annuli. We then quantified mitochondria within each annulus by recording pixel gray levels against a low and clearly separated background. We defined total mitochondrial intensity within each annulus as the sum of gray levels of mitochondrial material (M7510-positive) segmented from image background. ${ }^{28}$ We used a mixed effects analysis of covariance model for formal statistical inference. ${ }^{29,30}$ Our model included an overall intercept term, a BD versus control grouping factor, radial perinuclear distance, and a group by distance interaction. Radial distances were treated as nested within subjects, and mitochondrial density was re-expressed on the square root scale to conform more closely to the constant variance and Gaussian distribution assumptions of the general linear model. This morphological analysis of mitochondrial distribution will be referred to as the "bulls eye" method.

\section{Cell Profiler Analysis of Mitochondrial Distribution in Fibroblasts}

An independent morphometric distributional analysis was run using the software Cell Profiler ${ }^{31}$ (Broad Institute, Cambridge MA) on a subset of the BD and control fibroblast lines (4 BD and 4 Control lines) to determine the effect of lithium treatment on mitochondrial distribution. In Cell Profiler, three single images from five fields per line (24 to 80 cells per line), one from the nuclear channel stained with Hoechst, one from the phalloidin channel to mark cell edges, and one from the M7510 channel were loaded into the analysis software. To control for any difference in intensity of the raw M7510 images and to obtain an accurate measure of the distance from the middle of the nucleus to the pieces of the mitochondrial network images from this channel were pre-processed in Image J (National Institutes of Health, Bethesda MD) using the sharpen, find edges, and make binary commands as used in Koopman et al ${ }^{32}$ before loading images into Cell Profiler. The Cell Profiler Relate module was then used to measure the distance from the nucleus boundary as a metric of the extent of the mitochondrial network in each cell. This same analysis was also used to quantify the distribution of cytochrome $\mathrm{C}$ staining in fibroblasts.

\section{Lithium Treatment of Fibroblast Cultures}

Lithium carbonate $\left(\mathrm{LiCO}_{3}\right)$ (Sigma Aldrich, Allentown, PA), at levels chosen to match those typically seen in blood in treated patients, was added to fibroblast cultures plated at $0.8 \times 10^{4}$ cells per well. Media was changed every other day, and cells were treated with lithium for 5 days.

\section{TUNEL Staining for Detection of Apoptotic Changes}

TUNEL staining of fibroblast cultures plated at $2.5 \times 10^{4}$ cells per coverslip was done as in McPhie et al. ${ }^{26}$

\section{Measurement of ATP Levels in Fibroblasts}

Cells were plated at a density of $2.5 \times 10^{4}$ cells per well in 24-well plate. On the day after plating, media in the wells receiving serum starvation was changed to media containing $1.5 \%$ FBS and left for 24 hours before running the assay. Thirty minutes before the assay, $125 \mu \mathrm{mol} / \mathrm{L}$ $\mathrm{H}_{2} \mathrm{O}_{2}$ was added to the wells receiving serum starvation. ATP levels were measured with the ATPlite 1step kit (Perkin Elmer, Waltham MA) according to manufacturer's instructions. Three triplicate samples (for a total of nine readings) of each fibroblast line were measured for baseline ATP levels and ATP levels after the stress of serum starvation and $125 \mu \mathrm{mol} / \mathrm{L} \mathrm{H}_{2} \mathrm{O}_{2}$ treatment. 
A BD

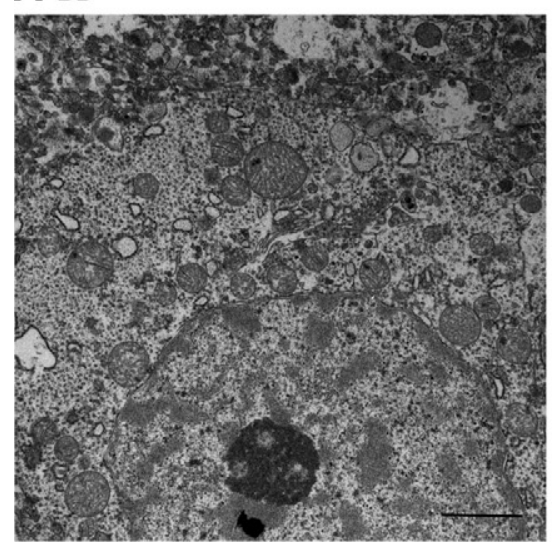

B control

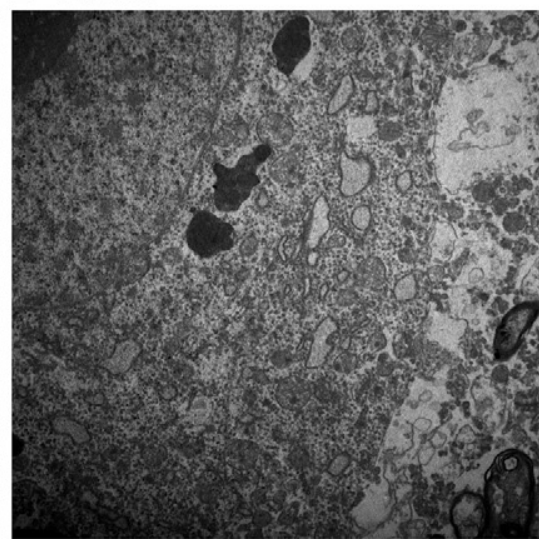

C

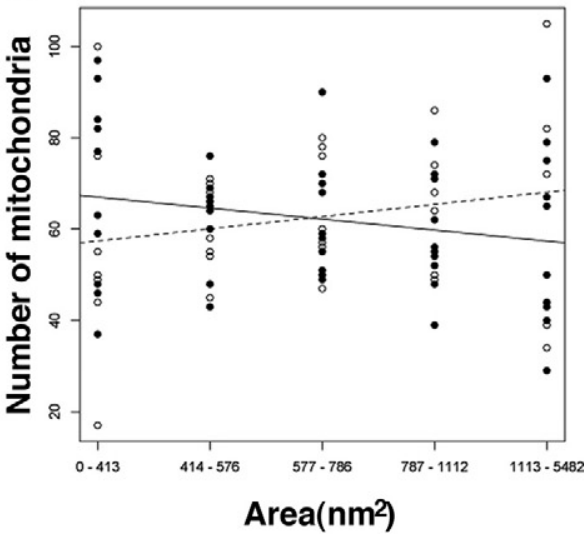

Figure 1. Neurons of patients with BD display alterations in mitochondrial area. Representative ultrastructural images of the prefrontal cortex of BD (A) and control brain (B). Scale bar $=2 \mu \mathrm{m}(\mathbf{A}$ and $\mathbf{B})$. C: Plot of the number of mitochondria in each of five quintiles of mitochondrial area in cells of PFC. Solid line and filled circles are BD. Dashed line and open circles are control.

\section{Cytochrome C Expression Levels and Intensity Correlation C (Colocalization) of M7510 Staining and Cytochrome C Immunocytochemistry}

Fibroblasts were plated on coverslips and labeled with M7510 and fixed as above. The cells were then probed with a specific antibody to cytochrome $\mathrm{C}$ as above without the steps of visualizing $\beta$ tubulin. Images taken at $\times 630$ were analyzed with Cell Profiler's Measure Object Intensity module, and integrated intensity levels of cytochrome $\mathrm{C}$ expression were compared between groups. Integrated intensity is defined as the sum of the pixel intensities within the mitochondrial network. A total of 295 control cells and $276 \mathrm{BD}$ cells were analyzed. All images were taken with the same exposure time. The Measure Correlation module was used to determine the degree of overlap between the two mitochondrial labels.

\section{Results}

\section{More Mitochondria of Smaller Area Are Observed in BD Brains}

Previous studies have indicated mitochondrial differences in volume density and numbers of mitochondria in oligodendroglia from postmortem brain in Schizophrenia. ${ }^{33}$ In this study we first asked whether abnormalities in mitochondrial morphology and size were present in postmortem BD brain. Prefrontal cortex was chosen, as it is a documented site of abnormalities in brain structure and function in patients with $\mathrm{BD} .{ }^{34}$ Ultrastructural examination of human brain tissue from healthy controls and $\mathrm{BD}$ brains showed that most mitochondria were small rounded structures of uniform size- 0.2 to $1.5 \mu \mathrm{m}$ in diameter with occasional rod-shaped profiles up to $2 \mu \mathrm{m}$ in length. A smooth outer membrane with invaginated inner cristae bound each organelle and small mitochondria profiles were seen interspersed with several larger profiles. Examples of tissue quality and mitochondrial profiles analyzed are illustrated in Figure 1, A and B. To quantify differences in mitochondrial size we measured mitochondrial area in electron micrographs from postmortem PFC of BD and control brains and binned the area measurements into equal quintiles. We found significantly smaller individual mitochondrial areas in the $\mathrm{BD}$ sample $(P=0.03$; $t$ test) (Figure $1 C$ ).

Because our sample size was small, we could not do a full analysis of drug effects on mitochondrial area. However, using a one-way analysis of variance to look at correlation with lithium treatment, one of the most common medications used for BD, no significant differences in mitochondrial area were seen between the four $\mathrm{BD}$ subjects on lithium versus the other BD subjects on other medications. Additionally, we did not see an association between mitochondrial area and age or postmortem delay (see supplemental Figure 1 at http://ajp.amjpathol.org).

\section{Cytochrome C Distribution in BD and Control Prefrontal Cortex}

We examined the samples for distributional differences of mitochondria in brain between the two groups. Previous studies by Kung and Roberts ${ }^{35}$ indicated mitochondrial distributional differences in the neuropil of the striatum of schizophrenic subjects. To assess this parameter in our BD samples, we used cytochrome $\mathrm{C}$ immunocytochemistry as a marker of mitochondria in postmortem brain tissue from the PFC. The analysis chosen, line profile analysis of stained cells in PFC, showed no significant differences between groups in mitochondrial distribution. In this analysis the cytochrome C pixel intensity curves varied greatly in shape across subjects in the BD group and across subjects in the control group. Although the two groups of curves appeared different from one another, subsequent comparison of the group-averaged pixel intensity curves yielded no significant differences in overall shape or at curve extrema $(P>0.30)$. 

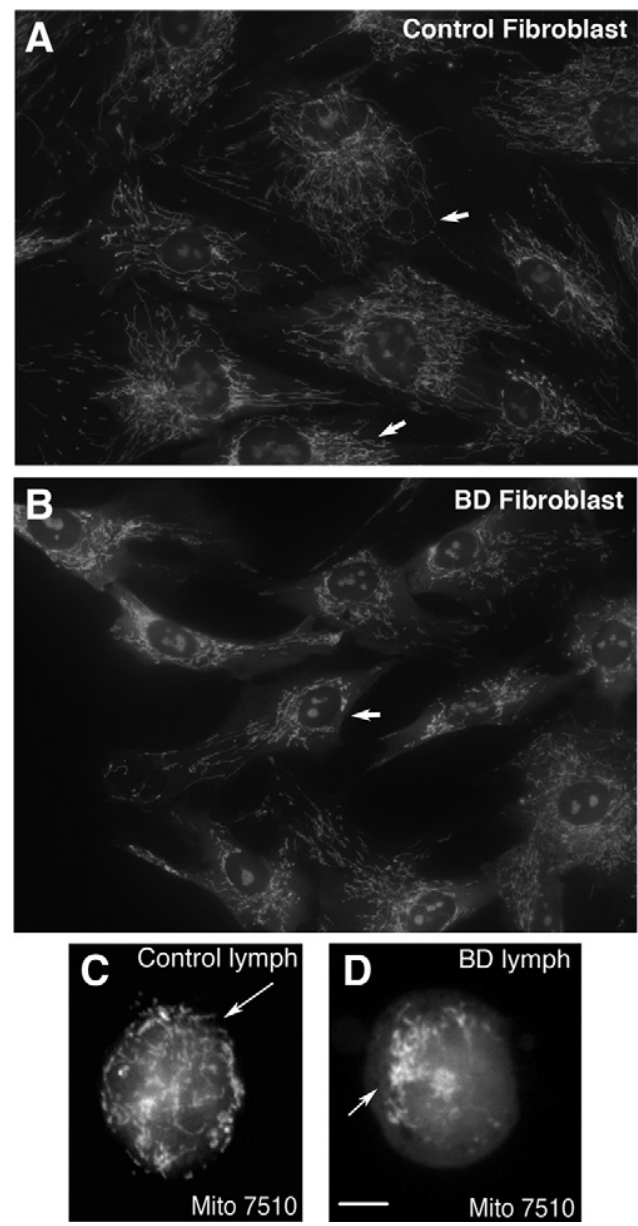

Figure 2. Mitochondrial clustering in primary cells from patients with BD. Compared with control cells (A, fibroblast, and $\mathbf{C}$, lymphocyte), primary fibroblasts (B) and lymphocytes (D) from patients with BD show altered mitochondrial morphology and distribution (arrows point to features to compare between cells, including the greater clustering of mitochondria around the nucleus and the relative paucity of mitochondria near the external cell membrane in BD). In the majority (greater than $70 \%$ ) of BD cells, perinuclear clusters, revealed using Mitotracker 7510 fluorescence, were observed. Magnification: $\times 630(\mathbf{A}$ and $\mathbf{B}), \times 1000(\mathbf{C}$ and $\mathbf{D})$. Scale bar $=10 \mu \mathrm{m}$.

\section{BD Fibroblasts and Lymphocytes Exhibit Alterations in Mitochondrial Morphology}

Although the identification of altered mitochondrial morphology (size) in the brain could be of value in studying various disease states, demonstrating consistent and reliable morphological abnormalities and a possible disease-specific relationship linked to mitochondrial function is difficult using postmortem brain tissue. This is due largely to variations in postmortem interval and fixation conditions. For these reasons, we selected two non-neuronal cell types for study - fibroblasts and lymphocytesharvested antemortem from individuals with BD. Using the mitochondrial-selective fluorescent marker, M7510, we found that fibroblasts (Figure 2A) and lymphocytes (Figure $2 \mathrm{C}$ ) from healthy controls displayed a highly organized and interconnected mitochondrial network that extended from the nuclear envelope to the plasma membrane. The network was composed of elements that ranged in shape from single profiles to interconnected stacks. A typical single mitochondrion was thin and straight with a relatively constant diameter with frequent branching. A few smaller sphere or cup-shaped profiles were observed. In contrast to control cells, the same cell types from patients with $\mathrm{BD}$ exhibited alterations in mitochondrial distribution and morphology. Mitochondria in the $\mathrm{BD}$ cell lines appeared as dense or bulky networks with perinuclear clustering, which was observed in greater than $70 \%$ of fibroblasts and lymphocytes in samples from BD patients of all ages (Figure 2, B and D). In addition to differences in the spatial distribution and clustering of the mitochondrial reticulum seen in the BD cells, we observed that mitochondria within the clusters appeared as thick, short segments or punctate, cup- or ring-shaped fluorescent profiles (see supplemental Figure 2, A-C at http://ajp.amjpathol.org). These clusters varied in size but were larger than those in control cells.

\section{Spatial Distribution of Mitochondria in Primary Cells from BD Patients}

We quantified the spatial variation in the perinuclear distribution of mitochondria using a segmentation algorithm. ${ }^{28}$ Cells from individuals with $\mathrm{BD}$ possessed higher mitochondrial densities at every radial distance from the nuclear envelope within the close, $15-70 \mu \mathrm{m}$, range relative to healthy controls (Figure 3, A-C). Also, our mixed effects ANCOVA model confirmed that for BD subjects the average mitochondrial density by radial perinuclear distance declined more steeply than that of the control group (BD group, $-1.15 / \mu \mathrm{m}$; control group, $-1.07 / \mu \mathrm{m}$, diagnosis by distance interaction $P=0.0008$ ) (Figure $3 C)$. We also measured cell area, cell length, cell width, and cell perimeter of the fibroblasts used in this analysis to verify that the observed mitochondrial distributional differences were not simply a result of BD cells being smaller, shorter, or fatter. No significant differences in any of these measurements were seen based on diagnosis after two sample $t$ tests.

\section{Morphometric Analysis of Fibroblast Mitochondria at the Ultrastructural Level}

Mitochondrial area, length, width, and aspect ratio were quantified using a two-dimensional analysis in our fibroblast samples. Although mitochondria in the BD samples showed a trend toward smaller mean area, as was seen in brain, this trend did not reach significance $(P>0.3)$. Quantification of the number of ring- and cup-shaped mitochondrial profiles showed that BD samples had more ring- and cup-shaped mitochondria (mean of 5.7 in 7 control lines and 8.5 in 8 BD lines; two-sample $t$ test, $P=0.034$ ). Examples of ring- and cup-shaped mitochondria are shown in supplemental Figure 2, D-H at http://ajp.amjpathol.org. 

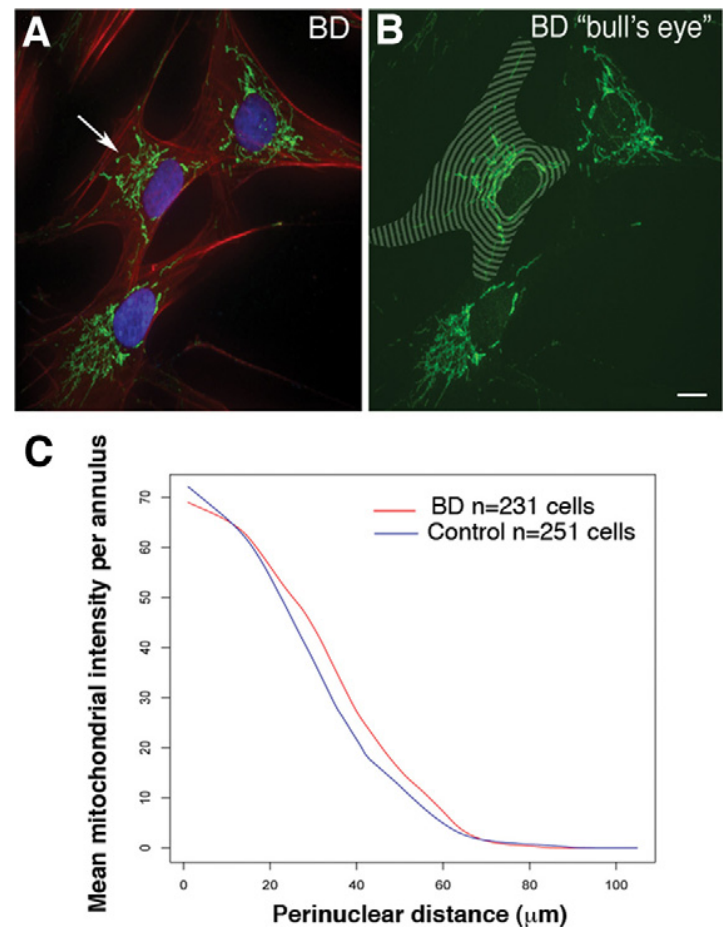

Figure 3. Analysis of mitochondrial distribution in cells from patients with BD. Morphometric analysis of mitochondrial spatial distribution, identified with Mitotracker 7510 (green), in human fibroblasts from normal controls ( $n=251$ cells $)$ and patients with $\mathrm{BD}(n=231$ cells). A representative cell from a BD patient is shown with an arrow pointing to the mitochondrial network (A). Concentric annuli in the shape of each nuclear envelope were placed to align with the centroids of each nucleus (B) to cover the spatial extent of each fibroblast. BD cells exhibit higher mitochondrial densities (square root scale) than those of the control group in the 15-70 $\mu \mathrm{m}$ range. Our random effects analysis of covariance of mitochondrial staining revealed that in the BD group of cells (red) there was a significantly steeper density decline than in the control group (blue) with distance from the nucleus $(\mathbf{C})$ (diagnosis by distance interaction $P=0.0008)$. Scale bar $=10 \mu \mathrm{m}(\mathbf{A}$ and $\mathbf{B})$.

\section{Lithium Effects on Mitochondrial Distribution in Fibroblasts}

Mood stabilizing drugs such as lithium are commonly used as treatments for $\mathrm{BD},{ }^{36,37}$ and all but one of our subjects with $\mathrm{BD}$ from whom fibroblasts were obtained were receiving lithium. Additionally, lithium treatment has been shown to have direct and indirect effects on mitochondria. $^{7,38,39}$ Therefore, we asked whether lithium exposure could promote changes in mitochondrial structure similar to those seen in primary BD cells or, alternatively, whether exposure to lithium could rescue the distributional abnormalities in BD cells making them look more like controls. An age-matched subset of our fibroblasts from both diagnostic groups (four BD and four Control) were treated acutely for 5 days with $1 \mathrm{mmol} / \mathrm{L} \mathrm{LiCO}_{3}$ (approximately the average serum level of lithium in patients). After distributional analysis we found that there was no significant effect of Li treatment in either group in a one-way analysis (or of a group by Li treatment in a two way analysis). There was, however, a significant difference in mitochondrial distribution between our two diagnostic groups (control versus $\mathrm{BD} ; P<0.05$, two-sample $t$ test) as observed in untreated cells (Figure 4, A versus C). The distributional analysis used for this part of the
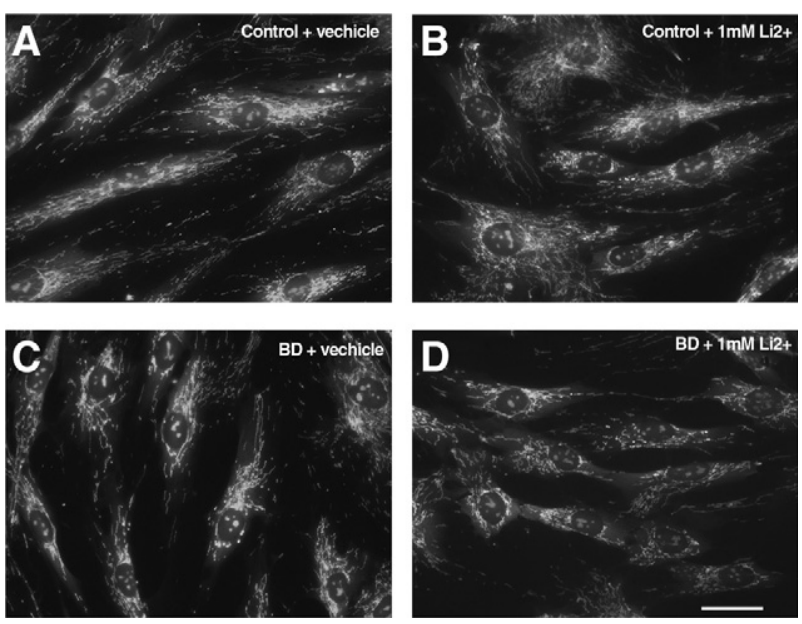

Figure 4. Lithium treatment does not cause mitochondrial abnormalities in control fibroblasts or rescue mitochondrial abnormalities in BD fibroblasts. Light microscopic examination of human fibroblasts, from psychiatricallyhealthy control and BD subjects, (B) and (D) or vehicle (A) and (C) each exposed to LiCO3 at $1 \mathrm{mmol} / \mathrm{L} 5$ days and labeled with M 7510. Analysis revealed no significant differences in mitochondrial shape and distribution compared with fibroblasts treated with vehicle only.

study, performed by Cell Profiler, provided an independent confirmation of disease-related differences observed in our bulls-eye analysis on a separate subset of cells from our BD and Control lines.

\section{Cytochrome C Distributional Differences in BD Fibroblasts}

To further characterize mitochondrial abnormalities seen in the BD fibroblasts, we asked whether the distribution of another component of mitochondria, cytochrome C, would also show differences in the BD lines. An agematched subset of fibroblasts (four BD and four Control) was labeled with an antibody to cytochrome C. The immunocytochemical staining of this antibody under our staining protocol allowed us to visualize very specific staining of the mitochondrial network in the fibroblasts. After automated distributional analysis with Cell Profiler we saw a significant difference in cytochrome $\mathrm{C}$ distribution in the BD cell lines versus the lines from healthy subjects (two-sample $t$ test, $P<0.0001$ ) (Figure 5). This result revealed that a separate component of the mitochondria also showed an altered distribution in the BD group, further confirming the mitotracker M7510 result.

\section{Cytochrome C Expression Levels and Intensity Correlation Analysis of M7510 Staining and Cytochrome C Immunocytochemistry}

To determine whether the level of expression of cytochrome $\mathrm{C}$ was different between the two experimental groups, we quantified the staining intensity of an antibody specific for cytochrome $\mathrm{C}$ in images of BD and Control fibroblasts by an automated analysis. No significant differences in cytochrome $\mathrm{C}$ immunocytochemical staining intensity were found $(20.61 \pm 5.5$ vs. $19.99 \pm 6.1$ Inten- 


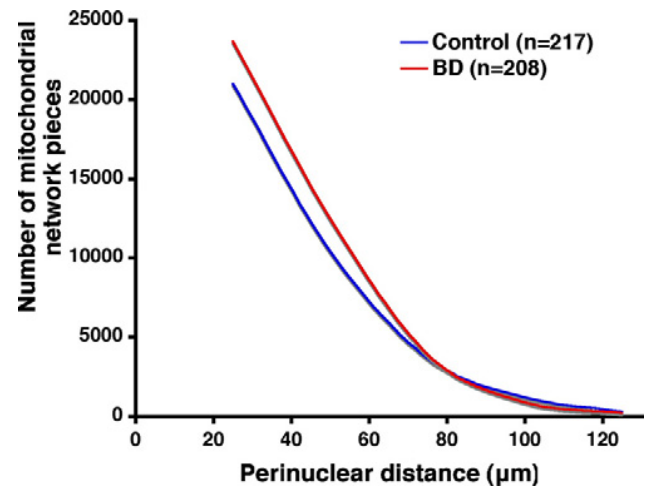

Figure 5. Cytochrome $\mathrm{C}$ expression shows an altered distribution in $\mathrm{BD}$ fibroblasts. Distributional analysis of cytochrome $\mathrm{C}$ immunocytochemistry demonstrated an altered distribution of the mitochondria network in BD fibroblasts as compared with Control fibroblasts. (two-sample $t$ test; $P<$ $0.0001)$.

sity Units; two-sample $t$ test NS; $P=0.63)$. The values are reported as intensity units because microscopes are not calibrated to an absolute scale. Because similar localization (distributional) changes were seen with both the $\mathrm{M} 7510$, a probe that is sequestered in the mitochondria and binds to proteins that have thiol moieties, and a specific antibody to cytochrome $\mathrm{C}$, we asked whether the two probes were labeling the same or different subsets of pieces of the mitochondrial network. Cell profiler automated intensity correlation analysis yielded a correlation coefficient of the two probes of 0.8 to 0.9 , indicating that both probes are labeling the same pieces of the network.

\section{ATP Measurement of BD and Control Fibroblasts at Baseline and after Oxidative Challenge}

Abnormal mitochondrial shape and distribution may be associated with abnormal oxidative phosphorylation and production of ATP. Testing for this possibility, we observed no significant differences in ATP levels between the two groups either at baseline or after serum starvation and $125 \mu \mathrm{mol} / \mathrm{L} \mathrm{H}_{2} \mathrm{O}_{2}$ challenge (see supplemental Figure 3 at $h$ ttp://ajp.amjpathol.org).

\section{Fibroblasts from Patients with BD Do Not Exhibit Substantial Cytoskeletal Alterations or Features of Apoptosis}

Mitochondria are known to move by closely associating with the cytoskeleton. We examined two key cytoskeletal proteins to see whether the mitochondrial distribution abnormalities might be a consequence of abnormalities in the cytoskeleton. Filamentous actin was examined by staining with phalloidin. Because mitochondria are directly associated with microtubules and microtubules have been demonstrated to have a direct role in mitochondrial biogenesis ${ }^{40}$ we examined the microtubule cytoskeleton with an antibody to tubulin $\beta$ l and $\beta \|$. Using F-actin selective phalloidin (red label, Figure 6, A-D) or antibodies to $\beta$ tubulin (red label, Figure $6, \mathrm{E}-\mathrm{H}$ ), we
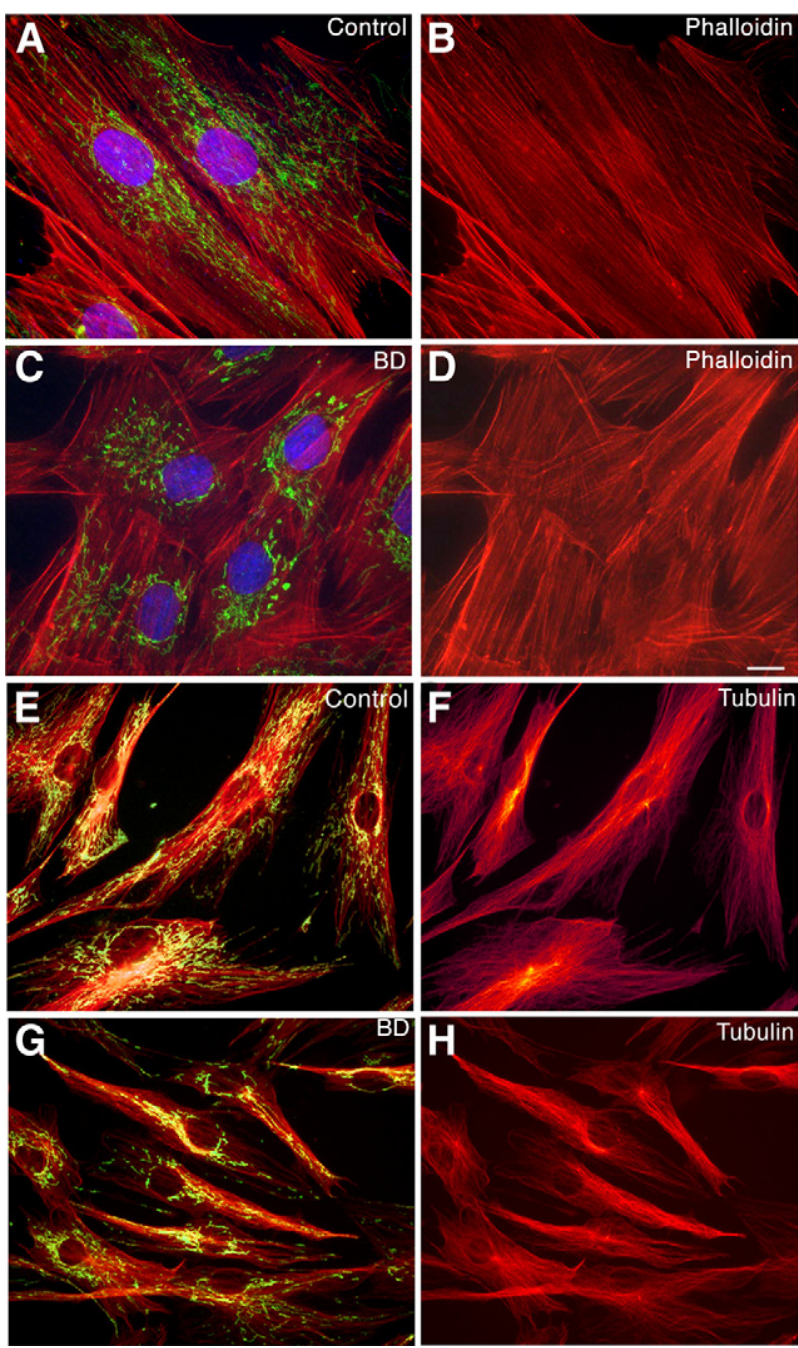

Figure 6. No substantial changes in the actin or tubulin cytoskeleton are seen that correlate with diagnosis in BD fibroblasts. Representative images of fibroblasts from patients with $\mathrm{BD}(\mathbf{C}, \mathbf{D}, \mathbf{G}$, and $\mathbf{H})$ and healthy controls (A, $\mathbf{B}, \mathbf{E}$, and $\mathbf{F}$ ) labeled with probes for mitochondria (M 7510 green $\mathbf{A}$ and $\mathbf{C}$, and orange $\mathbf{E}$ and $\mathbf{G}$ ), filamentous actin (red, phalloidin $\mathbf{A}-\mathbf{D}$ ), and tubulin $\beta$ (red, $\mathbf{E}-\mathbf{H})$. The results confirm mitochondrial redistribution in the BD fibroblasts compared with controls. However, abnormalities in mitochondrial shape and distribution were not accompanied by changes in the actin cytoskeleton, observed by phalloidin, or antibodies to tubulin $\beta \mathrm{I}$ and II, when compared with healthy controls. In addition, fluorescent staining of fibroblasts with the DNA-binding dyes, DAPI (A and $\mathbf{C}$ blue label) or bisbenzimide (not shown), revealed no evidence of increased apoptosis or nuclear fragmentation in the BD cells. Magnification: $\times 1000(\mathbf{A}-\mathbf{D}), \times 630$ (E-H). Scale bar $=10 \mu \mathrm{m}$

found that in BD cells, abnormalities in mitochondrial shape and distribution (Figure 6, green label C, yellowgreen label G) were not accompanied by substantial changes in the actin or tubulin cytoskeleton. Rather, BD cells showed a cytoskeletal pattern similar to that seen in healthy controls (Figure 6, B versus D, F versus $H$ ). In addition, DAPI labeling revealed no evidence of apoptosis or nuclear fragmentation in the BD cells (Figure 6C) compared with controls (Figure 6A). We also examined TUNEL staining of coverslips from the fibroblast samples. Counts of TUNEL-positive nuclei were not more than 5\% in any of our fibroblast samples, and no significant differences in TUNEL-positive nuclei between our BD and Control samples were seen. 


\section{Abnormalities of Other Membranous Organelles}

Inherited or environmentally induced abnormalities of mitochondria might affect other subcellular organelles, as well. However, in contrast to the altered cellular arrangement of the mitochondria in the BD cells, other organelles such as the plasma membrane, ER, and Golgi apparatus appeared similar to those in control cells (data not shown).

\section{Discussion}

To our knowledge, this study is the first to demonstrate that cells (neurons) in the prefrontal cortices of postmortem brain from patients with BD and peripheral cells from living BD patients display abnormalities in the morphology (size and shape) and intracellular distribution of mitochondria. We observed two different mitochondrial abnormalities in BD samples. In the PFC of BD brain, there were significantly more mitochondria of smaller size than in age-matched controls. In peripheral cells from BD patients, we quantified an altered distribution of the mitochondrial network such that there was a significantly faster drop off of mitochondria as one measures outward from the nucleus toward the cell periphery. This result was consistent between two independent mitochondrial probes. There were also more ring- or cup-shaped mitochondrial profiles in the BD fibroblast samples. Although the number of ring- and cup-shaped mitochondria counted in our sample is modest when compared with the total number of mitochondria counted, the BD samples had significantly more of these atypically shaped mitochondria, which may reflect subtle changes in the critical network architecture of mitochondria in the cells. Mitochondrial size, shape, and distribution typically differ between tissues, and the same factors may not cause exactly the same mitochondrial changes in different tissues. However, the abnormalities of mitochondrial size observed in BD brain may also be features of mitochondria in peripheral cells in BD, in which we saw a trend to abnormalities of size. Larger sample sizes than those available would be needed to confirm this difference. Similarly, the abnormalities of mitochondrial distribution observed in peripheral cells might also be features of mitochondrial networks in brain cells in BD. Although the analysis chosen for the present study was not able to detect a distributional difference, a more comprehensive study on a larger number of samples than those available for the present study might define disease-related mitochondrial distributional differences in the brain.

Alterations in mitochondrial shape and distribution could be the underlying cause of energy dysfunction in $\mathrm{BD}$, as observed in imaging studies, or may be secondary to other factors associated with BD. In either case, the mitochondrial abnormalities identified here may have implications for cell plasticity, resilience, and survival in patients with BD. They also may identify a pathognomic marker for BD or related illnesses and might, with further study, provide clues for studies targeted at the repair of the mitochondrial dysfunctions observed in BD.
Limitations of the results reported here include the modest number of tissue samples available. Our study also is limited in that only one brain region was examined with only a single analysis method. In addition, details of the medical history of the patients who provided the fibroblast samples, such as when in the disease the sample was taken, were not available. However, findings of alterations in mitochondria were consistent across three different tissues-brain, fibroblasts, and lymphocytes - from two different tissue collections. The size and distributional abnormalities observed were consistently seen in tissues from individuals with BD and were seen with a much lower frequency in psychiatrically healthy age-matched controls. Not all of the subjects were receiving the same medications, and the abnormality could not be replicated in control samples or rescued in BD samples by exposure of fibroblasts in vitro to the medication the subjects most commonly received (i.e., lithium). This suggests, but does not prove, that the effect is a corollary of illness rather than a consequence of treatment. Of course, even if the abnormality were drug related, it also would be of interest, as it might provide a clue to the mechanisms by which medications for BD act. Additionally, it is interesting that the mitochondrial abnormalities persist in vitro in cell lines and continue through many passages. A potential explanation for this is that the cell lines may carry genetic defects in nuclear or mitochondrial DNA similar to the mitochondrial DNA deletions seen by Kato et $\mathrm{al}^{4}$ in BD brains. Future work and nuclear and mitochondrial DNA sequencing may provide more information on this mechanism.

Evidence from a number of studies now shows that mitochondrial morphology is linked to energy metabolism and that changes in mitochondrial morphology reflect alterations in energy metabolism. Although the fibroblast lines in the present study did not show differences in ATP levels at baseline or with one level of oxidative challenge, a further or alternative stressor may bring out energy metabolism alterations. Alternatively, although an alteration in mitochondrial function would be consistent with our observations of altered mitochondrial location, it may also be that the only change present in the BD cells is a change in mitochondrial distribution. It has been suggested that mitochondrial distributional or localization changes alone may be the cause of psychiatric and other illnesses, in that the mitochondria in BD cells may not be able to deliver energy and other substrates to places in the cell that need it, thus leading to disease. Another point to be considered is that although we saw no differences in numbers of apoptotic cells in our samples, changes in mitochondrial distribution might render BD fibroblasts more sensitive or resistant to stress-induced apoptosis.

Mitochondria continually fuse and divide ${ }^{16-18}$ and the overall morphology of the mitochondrial network, which is prominent in eukaryotic cells, is modified by the balance of these two processes, ${ }^{41-43}$ which are regulated through the interactions of several well known mitochondrial GTPases. ${ }^{24,44-49}$ Deviation in either fission or fusion can result in cellular compromise leading to reduced cell function or even apoptosis and cell death. ${ }^{19,41,43,47,50,51}$ 
Mutations in genes encoding mitochondrial fission or fusion proteins have been associated with a number of disorders of the nervous system and can directly affect mitochondrial function. ${ }^{25,50,52,53}$ Overexpression of fusion proteins such as MFN2 or the down-regulation of fission proteins such as DRP1, affects mitochondrial function by reducing mitochondrial respiration and ATP levels and affects mitochondrial morphology leading to decreased mitochondrial branching, spheroid mitochondrial tubules, and ring-shaped mitochondria and fragmentation. ${ }^{19,20,22,23,41-43,47,50}$ G72 a candidate BD susceptibility gene has also been shown to affect mitochondrial fragmentation. ${ }^{54}$ Since one explanation for the increased numbers of smaller mitochondria seen in $\mathrm{BD}$ PFC is increased fragmentation, G72 or related proteins may play a role. There is also an ever growing set of studies indicating that dysregulation of mitochondrial fission and fusion may be important causative factors in the neurodegeneration seen in Alzheimer's, Parkinson's and Huntington's disease. ${ }^{51,55,56}$

Given the influence of fission and fusion events in mitochondrial structure, alterations in these processes might explain our findings of abnormal mitochondrial morphology and distribution in patients with $\mathrm{BD}$ and are appropriate targets for further study. Alternatively, a failure of mitochondrial transport due to a disruption in cytoskeletal proteins (i.e., actin, tubulin, or motor proteins such as kinesin ${ }^{57}$ ) could contribute to altered mitochondrial clustering. Although our examination of the cytoskeleton in primary fibroblasts from patients with BD did not show any substantial changes in cytostructure, a more detailed study centered on cytoskeletal proteins may shed light on more subtle alterations between the groups.

Cumulatively, our findings indicate that changes in mitochondrial shape and distribution are a consistent feature of neurons and primary non-neuronal cells from individuals with BD. Furthermore, our data are consistent with evidence from MRI ${ }^{9,10}$ and postmortem ${ }^{4-8}$ and genetic $^{4-8,11-15}$ studies linking reduced energy production and mitochondrial dysfunction to BD. Because of the well-characterized link between mitochondrial structure and function, our current findings of mitochondrial structural changes in cells from patients with BD support the hypothesis that there are abnormalities of mitochondria in BD. Specifically, our findings support the possibility that alterations in mitochondrial structure and function may underlie abnormal brain development or activation in BD. As mitochondria are crucial for cell growth, function, and survival, they may also play a role in the neuronal and/or glial compromise or cell deficits seen in BD. The peripheral cell models of $\mathrm{BD}$ described here may prove valuable in further defining the fundamental relationships between mitochondrial structure and function in $\mathrm{BD}$, examining how these interactions influence energy metabolism and elucidating molecular changes contributing to the expression of $\mathrm{BD}$. In time, further study of the phenomena observed could lead to the identification of specific pathognomic and pathophysiologic features of $\mathrm{BD}$, useful for diagnosis and for the design of treatments targeted specifically to the abnormalities that underlie BD.

\section{References}

1. Berns GS, Nemeroff CB: The neurobiology of bipolar disorder. Am J Med Genet C Semin Med Genet 2003, 123C:76-84

2. Rajkowska G: Cell pathology in bipolar disorder. Bipolar Disord 2002, 4:105-116

3. Quiroz JA, Gray NA, Kato T, Manji HK: Mitochondrially mediated plasticity in the pathophysiology and treatment of bipolar disorder. Neuropsychopharmacology 2008, 33:2551-2565

4. Kato T, Stine OC, McMahon FJ, Crowe RR: Increased levels of a mitochondrial DNA deletion in the brain of patients with bipolar disorder. Biol Psychiatry 1997, 42:871-875

5. Konradi C, Eaton M, MacDonald ML, Walsh J, Benes FM, Heckers S: Molecular evidence for mitochondrial dysfunction in bipolar disorder. Arch Gen Psychiatry 2004, 61:300-308

6. Iwamoto K, Bundo M, Kato T: Altered expression of mitochondriarelated genes in postmortem brains of patients with bipolar disorder or schizophrenia, as revealed by large-scale DNA microarray analysis. Hum Mol Genet 2005, 14:241-253

7. Sun X, Wang JF, Tseng M, Young LT: Downregulation in components of the mitochondrial electron transport chain in the postmortem frontal cortex of subjects with bipolar disorder. J Psychiatry Neurosci 2006, 31:189-196

8. Munakata K, Iwamoto K, Bundo M, Kato T: Mitochondrial DNA $3243 A>G$ mutation and increased expression of LARS2 gene in the brains of patients with bipolar disorder and schizophrenia. Biol Psychiatry 2005, 57:525-532

9. Keener MT, Phillips ML: Neuroimaging in bipolar disorder: a critical review of current findings. Curr Psychiatry Rep 2007, 9:512-520

10. Frey BN, Stanley JA, Nery FG, Monkul ES, Nicoletti MA, Chen HH, Hatch JP, Caetano SC, Ortiz O, Kapczinski F, Soares JC: Abnormal cellular energy and phospholipid metabolism in the left dorsolateral prefrontal cortex of medication-free individuals with bipolar disorder: an in vivo 1H MRS study. Bipolar Disord 2007, 9 (Suppl 1):119-127

11. Benes FM, Matzilevich D, Burke RE, Walsh J: The expression of proapoptosis genes is increased in bipolar disorder, but not in schizophrenia. Mol Psychiatry 2006, 11:241-251

12. Naydenov AV, MacDonald ML, Ongur D, Konradi C: Differences in lymphocyte electron transport gene expression levels between subjects with bipolar disorder and normal controls in response to glucose deprivation stress. Arch Gen Psychiatry 2007, 64:555-564

13. Kato T, Kato N: Mitochondrial dysfunction in bipolar disorder. Bipolar Disord 2000, 2:180-190

14. Washizuka S, Kakiuchi C, Mori K, Tajima O, Akiyama T, Kato T: Expression of mitochondria-related genes in lymphoblastoid cells from patients with bipolar disorder. Bipolar Disord 2005, 7:146-152

15. Munakata K, Tanaka M, Mori K, Washizuka S, Yoneda M, Tajima O, Akiyama T, Nanko S, Kunugi H, Tadokoro K, Ozaki N, Inada T, Sakamoto K, Fukunaga T, lijima Y, Iwata N, Tatsumi M, Yamada K, Yoshikawa T, Kato T: Mitochondrial DNA 3644T->C mutation associated with bipolar disorder. Genomics 2004, 84:1041-1050

16. Escobar-Henriques M, Langer T: Mitochondrial shaping cuts. Biochim Biophys Acta 2006, 1763:422-429

17. Logan DC: The mitochondrial compartment. J Exp Bot 2006, 57:1225-1243

18. McBride HM, Neuspiel M, Wasiak S: Mitochondria: more than just a powerhouse. Curr Biol 2006, 16:R551-560

19. Bossy-Wetzel E, Barsoum MJ, Godzik A, Schwarzenbacher R, Lipton SA: Mitochondrial fission in apoptosis, neurodegeneration and aging. Curr Opin Cell Biol 2003, 15:706-716

20. Cerveny KL, Tamura Y, Zhang Z, Jensen RE, Sesaki H: Regulation of mitochondrial fusion and division. Trends Cell Biol 2007, 17:563-569

21. Koopman WJ, Verkaart S, Visch HJ, van der Westhuizen FH, Murphy MP, van den Heuvel LW, Smeitink JA, Willems PH: Inhibition of complex I of the electron transport chain causes O2-. -mediated mitochondrial outgrowth. Am J Physiol Cell Physiol 2005, 288:C1440-C1450

22. Mannella CA: The relevance of mitochondrial membrane topology to mitochondrial function. Biochim Biophys Acta 2006, 1762:140-147

23. Santel A: Get the balance right: mitofusins roles in health and disease. Biochim Biophys Acta 2006, 1763:490-499

24. Hermann GJ, Thatcher JW, Mills JP, Hales KG, Fuller MT, Nunnari J, Shaw JM: Mitochondrial fusion in yeast requires the transmembrane GTPase Fzo1p. J Cell Biol 1998, 143:359-373 
25. Zanna C, Ghelli A, Porcelli AM, Karbowski M, Youle RJ, Schimpf S, Wissinger B, Pinti M, Cossarizza A, Vidoni S, Valentino ML, Rugolo M, Carelli V: OPA1 mutations associated with dominant optic atrophy impair oxidative phosphorylation and mitochondrial fusion. Brain 2008, 131:352-367

26. McPhie DL, Coopersmith R, Hines-Peralta A, Chen Y, Ivins KJ, Manly SP, Kozlowski MR, Neve KA, Neve RL: DNA synthesis and neuronal apoptosis caused by familial Alzheimer disease mutants of the amyloid precursor protein are mediated by the p21 activated kinase PAK3. J Neurosci 2003, 23:6914-6927

27. Cleveland W: LOWNESS: a program for smoothing scatterplots by robust locally weighted regression. Am Stat 1981, 35:54

28. Young D, Ma J, Cherkerzian S, Froimowitz MP, Ennulat DJ, Cohen BM, Evans ML, Lange N: Automated identification of Fos expression. Biostatistics 2001, 2:351-364

29. Lange N, Laird NM: The effect of covarience structure on varience estimation in balanced growth-curve models with random parameters. J Am Statis Assoc 1989, 84:241-247

30. Venables WN RB: Statistics and computing: modern applied statistics with S. 2002, 512p

31. Carpenter AE, Jones TR, Lamprecht MR, Clarke C, Kang IH, Friman O, Guertin DA, Chang JH, Lindquist RA, Moffat J, Golland P, Sabatini DM: CellProfiler: image analysis software for identifying and quantifying cell phenotypes. Genome Biol 2006, 7:R100

32. Koopman WJ, Visch HJ, Smeitink JA, Willems PH: Simultaneous quantitative measurement and automated analysis of mitochondrial morphology, mass, potential, and motility in living human skin fibroblasts. Cytometry A 2006, 69:1-12

33. Uranova N, Orlovskaya D, Vikhreva O, Zimina I, Kolomeets N, Vostrikov V, Rachmanova V: Electron microscopy of oligodendroglia in severe mental illness. Brain Res Bull 2001, 55:597-610

34. Ongur D, Drevets WC, Price JL: Glial reduction in the subgenual prefrontal cortex in mood disorders. Proc Natl Acad Sci USA 1998, 95:13290-13295

35. Kung L, Roberts RC: Mitochondrial pathology in human schizophrenic striatum: a postmortem ultrastructural study. Synapse 1999 31:67-75

36. Friedman SD, Dager SR, Parow A, Hirashima F, Demopulos C, Stol AL, Lyoo IK, Dunner DL, Renshaw PF: Lithium and valproic acid treatment effects on brain chemistry in bipolar disorder. Biol Psychiatry 2004, 56:340-348

37. Goodwin FK, Fireman B, Simon GE, Hunkeler EM, Lee J, Revicki D: Suicide risk in bipolar disorder during treatment with lithium and divalproex. J Am Med Assoc 2003, 290:1467-1473

38. Shalbuyeva N, Brustovetsky T, Brustovetsky N: Lithium desensitizes brain mitochondria to calcium, antagonizes permeability transition, and diminishes cytochrome C release. J Biol Chem 2007, 282:18057-18068

39. Struewing IT, Barnett CD, Tang T, Mao CD: Lithium increases PGC1alpha expression and mitochondrial biogenesis in primary bovine aortic endothelial cells. FEBS J 2007, 274:2749-2765

40. Karbowski M, Spodnik JH, Teranishi M, Wozniak M, Nishizawa Y, Usukura J, Wakabayashi T: Opposite effects of microtubule-stabilizing and microtubule-destabilizing drugs on biogenesis of mitochondria in mammalian cells. J Cell Sci 2001, 114:281-291

41. Detmer SA, Chan DC: Functions and dysfunctions of mitochondrial dynamics. Nat Rev Mol Cell Biol 2007, 8:870-879
42. Knott AB, Perkins G, Schwarzenbacher R, Bossy-Wetzel E: Mitochondrial fragmentation in neurodegeneration. Nat Rev Neurosci 2008, 9:505-518

43. Plecita-Hlavata L, Lessard M, Santorova J, Bewersdorf J, Jezek P: Mitochondrial oxidative phosphorylation and energetic status are reflected by morphology of mitochondrial network in INS-1E and HEP-G2 cells viewed by 4Pi microscopy. Biochim Biophys Acta 2008, 1777:834-846

44. Cipolat S, Martins de Brito O, Dal Zilio B, Scorrano L: OPA1 requires mitofusin 1 to promote mitochondrial fusion. Proc Natl Acad Sci USA 2004, 101:15927-15932

45. Frederick RL, McCaffery JM, Cunningham KW, Okamoto K, Shaw JM: Yeast Miro GTPase. Gem1p, regulates mitochondrial morphology via a novel pathway. J Cell Biol 2004, 167:87-98

46. Lee S, Jeong SY, Lim WC, Kim S, Park YY, Sun X, Youle RJ, Cho H: Mitochondrial fission and fusion mediators, hFis1 and OPA1, modulate cellular senescence. J Biol Chem 2007, 282:22977-22983

47. Santel A, Fuller MT: Control of mitochondrial morphology by a human mitofusin. J Cell Sci 2001, 114:867-874

48. Stojanovski D, Koutsopoulos OS, Okamoto K, Ryan MT: Levels of human Fis1 at the mitochondrial outer membrane regulate mitochondrial morphology. J Cell Sci 2004, 117:1201-1210

49. Stojanovski D, Rissler M, Pfanner N, Meisinger C: Mitochondrial morphology and protein import-a tight connection? Biochim Biophys Acta 2006, 1763:414-421

50. Frank S: Dysregulation of mitochondrial fusion and fission: an emerging concept in neurodegeneration. Acta Neuropathol 2006 111:93-100

51. Knott AB, Bossy-Wetzel E: Impairing the mitochondrial fission and fusion balance: a new mechanism of neurodegeneration. Ann NY Acad Sci 2008, 1147:283-292

52. Davies VJ, Hollins AJ, Piechota MJ, Yip W, Davies JR, White KE, Nicols PP, Boulton ME, Votruba M: Opa1 deficiency in a mouse model of autosomal dominant optic atrophy impairs mitochondrial morphology, optic nerve structure and visual function. Hum Mol Genet 2007, 16:1307-1318

53. Olichon A, Baricault L, Gas N, Guillou E, Valette A, Belenguer P, Lenaers G: Loss of OPA1 perturbates the mitochondrial inner membrane structure and integrity, leading to cytochrome $\mathrm{c}$ release and apoptosis. J Biol Chem 2003, 278:7743-7746

54. Kvajo M, Dhilla A, Swor DE, Karayiorgou M, Gogos JA: Evidence implicating the candidate schizophrenia/bipolar disorder susceptibility gene G72 in mitochondrial function. Mol Psychiatry 2008, 13:685-696

55. Lutz AK, Exner N, Fett ME, Schlehe JS, Kloos K, Lammermann K, Brunner B, Kurz-Drexler A, Vogel F, Reichert AS, Bouman L, VogtWeisenhorn D, Wurst W, Tatzelt J, Haass C, Winklhofer KF: Loss of parkin or PINK1 function increases Drp1-dependent mitochondrial fragmentation. J Biol Chem 2009, 284:22938-22951

56. Wang X, Su B, Lee HG, Li X, Perry G, Smith MA, Zhu X: Impaired balance of mitochondrial fission and fusion in Alzheimer's disease. J Neurosci 2009, 29:9090-9103

57. De Vos KJ, Allan VJ, Grierson AJ, Sheetz MP: Mitochondrial function and actin regulate dynamin-related protein 1-dependent mitochondrial fission. Curr Biol 2005, 15:678-683 